\title{
THE CLINICAL SIGNIFICANCE OF DYSLIPIDAEMIAS
}

\author{
Alan Taylor, FRCPath
}

\begin{abstract}
Most people are aware that cholesterol in the blood can be 'high' or 'low'. Those who are better informed are aware that the serum cholesterol is contained in two fractions, one of which is damaging to health and another which is beneficial. Fewer realize that there are six types of cholesterol containing particles which promote the formation of atheroma, the hard muco-fatty material within the intima of the larger arteries, and only one which confers protection against this. These particles are complex structures containing cholesterol, cholesterol esters, phospholipid and triglyceride. In this article, Dr Alan Taylor, Consultant in Chemical Pathology at Furness General Hospital and Honorary Lecturer in Biochemistry at Lancaster University, describes the work of the specialist Lipid Clinic and gives us an insight into the detailed and complex relationships between the lipid particles and disease, particularly coronary heart disease (CHD).
\end{abstract}

\section{- Units throughout are in mmol/l except where stated.}

Lipoprotein particles can be separated by the analytical ultracentrifuge in accordance with their densities. Lipoproteins are labeled as very low (VLDL), intermediate (IDL), low (LDL) and high (HDL) densities. The lower density particles contribute to atherosclerotic plaques and destabilize them. HDL is unique in that it reduces and stabilizes them.

Table 1 shows a reasonably comprehensive list of lipid abnormalities, both primary or genetic and secondary to other disorders. Table 2 shows those causing accelerated atherogenesis especially in those with other risk factors. Table 3 shows conditions in which there is protection against atheroma; hyperolipoproteinaemia is a condition in which longevity of family members is common and cannot be described as a disease. Some disorders of lipid metabolism have a tendency to provoke autolysis of the pancreas, and may show hepatomegaly and peripheral neuropathy. Others are associated with deficiencies of fat-soluble vitamins.

The reason for the abnormalities can only be appreciated by knowing how lipids are metabolized. For clarity, exogenous processes are considered separately from endogenous ones.

\section{Lipid metabolism:}

a) exogenous - gut

After a meal containing fat, which is principally triglyceride (TG), digestion produces fatty acids which are re-esterified in enterocytes and packaged there in large particles called chylomicrons. They make the serum look turbid and we describe the appearance as 'lipaemic'. On standing, they rise to form a white layer on top. Clearance from the portal blood is hepatic and extrahepatic by membrane lipases as described below for VLDL. The serum is cleared by many individuals within four hours and the serum triglyceride decays to the normal fasting values of between 0.4 and $1.7 \mathrm{mmol} / \mathrm{l}$.

Of the cholesterol absorbed from the small bowel, a third is derived from diet and the rest is from bile. The bile acids are formed from cholesterol and there is some reabsorption of cholesterol with these at the terminal ileum. There is no increase in cholesterol after meals because of the large contribution from the enterohepatic circulation.

\section{b) endogenous - liver}

Between meals, VLDL particles are formed in the liver. They contain TG predominantly and a small amount of cholesterol and protein. In the sub-nourished individual the TG provides fuel for cells and in the well-fed it is destined for storage in adipose tissue.

Lipoprotein lipase (LPL) is an enzyme which is loosely bound to vascular endothelial surfaces. It removes TG from the particle leaving IDL; this becomes bound to receptors in the liver by one of its proteins called apolipoprotein E. This IDL is modified and is catabolized so rapidly in normals that little exists in the circulation. Both hepatic lipase and LPL are involved in its metabolism continuing, the TG-clearing to produce LDL. This binds to specific LDL-receptors on cells or is taken up by macrophages. Lipid exchange between particles is dynamic and occurs between all the lipoprotein particles.

The metabolism of lipoproteins is completed by HDL, which takes free cholesterol from oxidized LDL in tissues and esterifies it for catabolism or excretion into the bile.

It is important to recognize that the processing of $\mathrm{TG}$ is at a rate vastly greater than that of LDL-cholesterol.

Reviews are available to describe in great detail the normal and abnormal processes and their significance ${ }^{(1.2)}$. I will present a few cases as examples of abnormalities which produce clinical problems.

\section{CASE REPORTS}

Case 1: an uncomplaining man

On screening a 45-year-old man working on nuclear submarines, a blood film showed acanthocytosis (Figure 1). He had no complaints but on questioning had regularly had three pale-coloured motions a day for years. The fact that he played darts and golf seemed to preclude any neurological abnormality. Serum Vitamins A, D and K (prothrombin time) were normal, but the vitamin $\mathrm{E}$ was only just detectable.

His serum cholesterol was $1.0 \mathrm{mmol} / \mathrm{l}$ of which $0.8 \mathrm{mmol} / \mathrm{l}$ was HDL-associated. He had no detectable LDL-cholesterol and its principal apoprotein (apoB) was not detected by immunoassay. The two hypolipidaemias possible are 


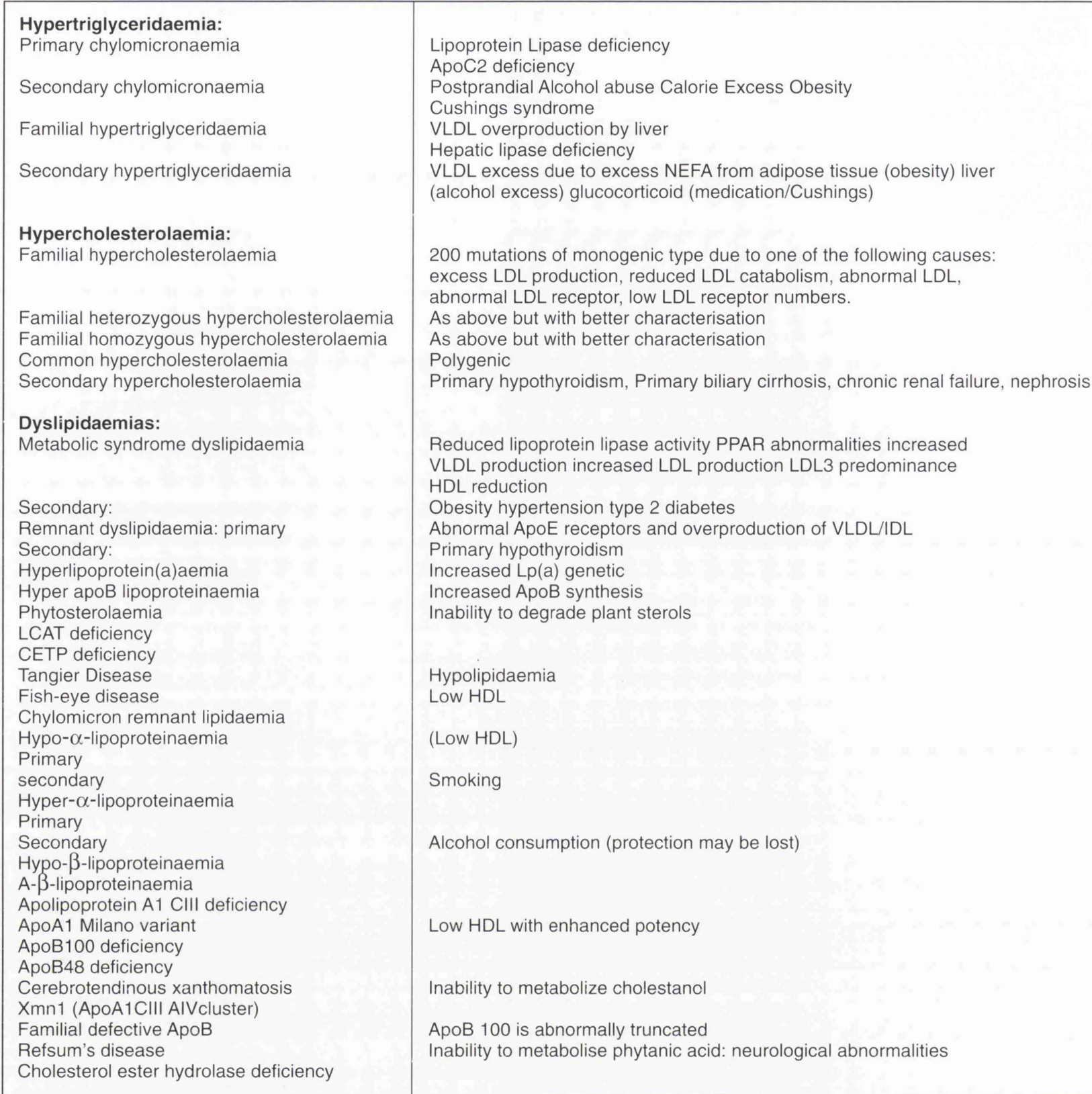

Table I Disorders of lipid metabolism and their causes

- Remnant lipidaemia (dys- $\beta$-lipoproteinaemia)[Frederickson type III]

- Familial combined hyperlipidaemia

- Hypertriglyceridaemia with hypo- $\alpha$-lipoproteinaemia

- Familial hypercholesterolaemia combined with any secondary dyslipidaemia

- Common hypercholesterolaemia combined with any secondary dyslipidaemia

- Hyper- $\beta$-lipoproteinaemia

- Secondary dyslipidaemia induced by: alcohol, calories, obesity, type II diabetes, hypothyroidism, nephrosis, liver disease, porphyria, anorexia nervosa, myeloma, drugs: Cyclosporin A, anti-psychotics, high dose bendrofluazide, some $\beta$-blockers (not bisprolol and not $<50 \mathrm{mg} / \mathrm{d}$ atenolol)

- Hypo- $\alpha$-lipoproteinaemia due to smoking

Table 2 Dyslipidaemias with CHD risk

a- $\beta$-lipoproteinaemia in which primary relatives have normal apoB levels and hypo- $\beta$-lipoproteinaemia in which they are about half-normal. The diagnosis on family studies was shown to be hypo- $\beta$-lipoproteinaemia. Vitamin E repletion is not usually advised in such cases but because of the severity it was felt to be advisable here.

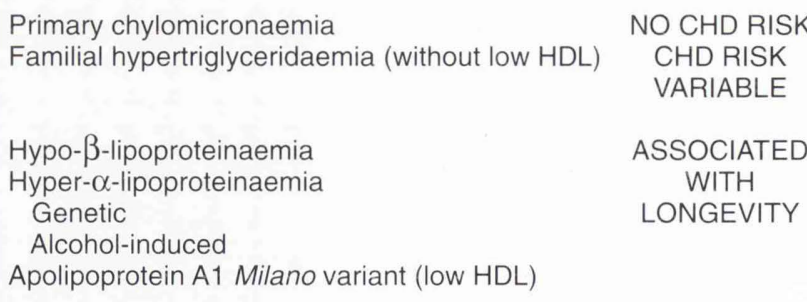

Hypo- $\beta$-lipoproteinaemia

Hyper- $\alpha$-lipoproteinaemia

Genetic

Alcohol-induced

Apolipoprotein A1 Milano variant (low HDL)

WITH

LONGEVITY

Table 3 Dyslipidaemias without CHD risk

Case 2: a 38-year-old mother with a high cholesterol A 38-year-old woman had a serum cholesterol 8.8, LDL 6.7, HDL 1.8 and normal FTG. Her brother, aged 40, was on statin therapy for primary prevention of heart disease. She gave no history of angina and had no conventional risk factors, being normotensive and a nonsmoker. She was 


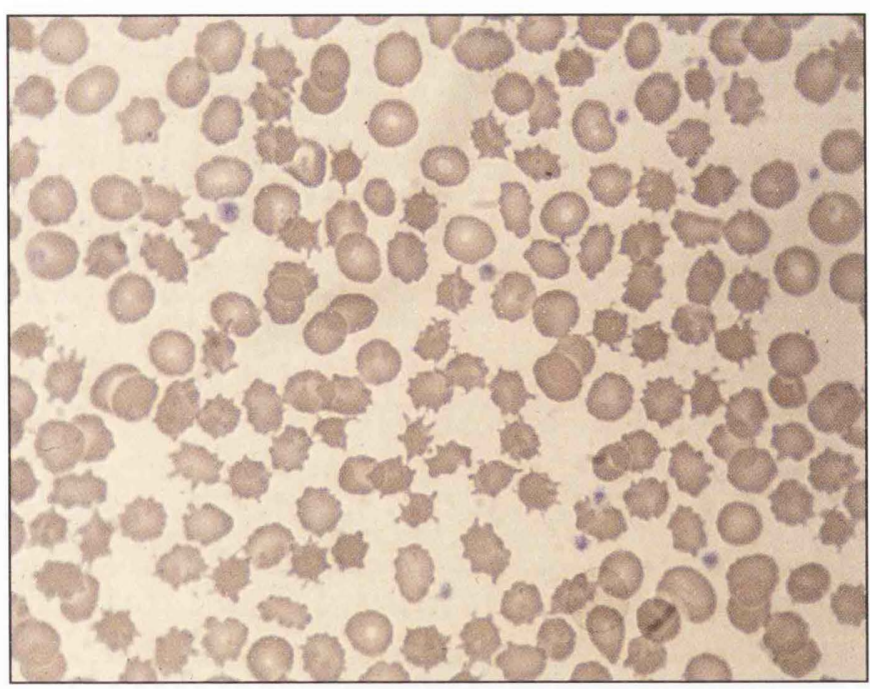

Figure 1 Routine blood film from a nuclear industry worker. The acanthocytosis is easily seen. In this case it was due to a dyslipidaemia.

euthyroid. An Lp(a) was measured at $400 \mathrm{mg} / \mathrm{dl}$. An increased risk is expected where this is over $30 \mathrm{mg} / \mathrm{dl}$.

An exercise ECG was arranged to see if she had occult heart disease and on the day before this she developed classic symptoms of angina. Coronary angiograms showed narrowing of the coronary tree but no stenoses worthy of bypass.

Her LDL is now reduced from $6.7 \mathrm{mmol} / 1$ to $2.9 \mathrm{mmol} / \mathrm{l}$ on statin therapy. This effectively reduces the risk from the high $L p(a) . L p(a)$ is an atherogenic lipoprotein associated with LDL and the treatment of this condition is LDL reduction with statin.

The diagnosis is familial hypercholesterolaemia and hyperlipoprotein(a)aemia. The former can be due to abnormal LDL receptors or reduced LDL receptor number.

From risk tables (Framingham) the risk of $\mathrm{CHD}$ at presentation was $2 \%$ over 10 years. Primary prevention is not always recommended for young females with high HDL. Her risk only became apparent when the $\mathrm{Lp}(\mathrm{a})$ fraction was measured, and was reduced by statin therapy.

\section{Case 3: a man on the waiting list}

A 45 -year-old man with a fasting TG of $14 \mathrm{mmol} / \mathrm{l}$ was referred to the Lipid Clinic. At referral, I sent a letter in response advising immediate dietary review and fibrate therapy. The patient was seen in due course one month after a hospital admission with abdominal pain. He had been diagnosed as acute pancreatitis and had not started the recommended therapy. He is now well on fibrate therapy three years later with no further attacks.

Acute pancreatitis may occur recurrently in those with high VLDL or in chylomicronaemia syndromes. Such patients may have hepatosplenomegaly and peripheral neuropathy. TG values over $11 \mathrm{mmol} / \mathrm{l}$, which are referred to as 'severe hypertriglyceridaemia', are those most at risk. Primary aetiologies are recognized but there are cases in the literature where the causes are 'extreme diets', eg consumption of several litres of ice cream a day. High serum TG is associated with increases in factor VII which may predispose to thrombotic events as well as atheroma formation.

\section{Practice Point}

Hypertriglyceridaemia may cause acute pancreatitis. Anorexia as a consequence of this involves a prolonged fast during which hypertriglyceridaemia may resolve. This is analogous to pancreatitis secondary to hypercalcaemia which may also be missed because of soap formation in tissues and consequent hypocalcaemia at presentation.

Case 4: an inadequately-treated lipidaemia

A 60-year-old male nonsmoker with hypertension, whose mother died suddenly aged 60 , is known to have familial combined hyperlipidaemia. His BP is $170 / 90$ on treatment. $\mathrm{He}$ had lost weight on diet but was less than adequately controlled on statin.

$\begin{array}{cccccl}\text { TC } & \text { LDL } & \text { HDL } & \text { FTG } & \text { Risk } & \\ 9.1 & 6.5 & 1.1 & 3.2 & 8.3 & \text { initial } \\ 6.7 & 3.6 & 1.1 & 4.5 & 5.9 & \text { on diet and statin (nocte) }\end{array}$

The addition of a small dose (200mg) bezafibrate (mane), a second-generation fibrate which lowers both LDL and TG/HDL ratio produced within four weeks, a significant reduction in risk:

\section{TC LDL HDL FTG Risk

$\begin{array}{lllll}4.3 & 2.0 & 1.3 & 2.1 & 3.2\end{array}$

This shows how combination therapy can work in the individual. He meets the Joint British Guidelines (JBS) current criteria for LDL reduction ${ }^{(3)}$ and the American Heart Association (AHA) guidelines on HDL increase ${ }^{(4)}$. His FTG is not quite at the AHA-approved level of below $1.7 \mathrm{mmol} / \mathrm{l}$, which is the level at which the atherogenic LDL III fraction is low.

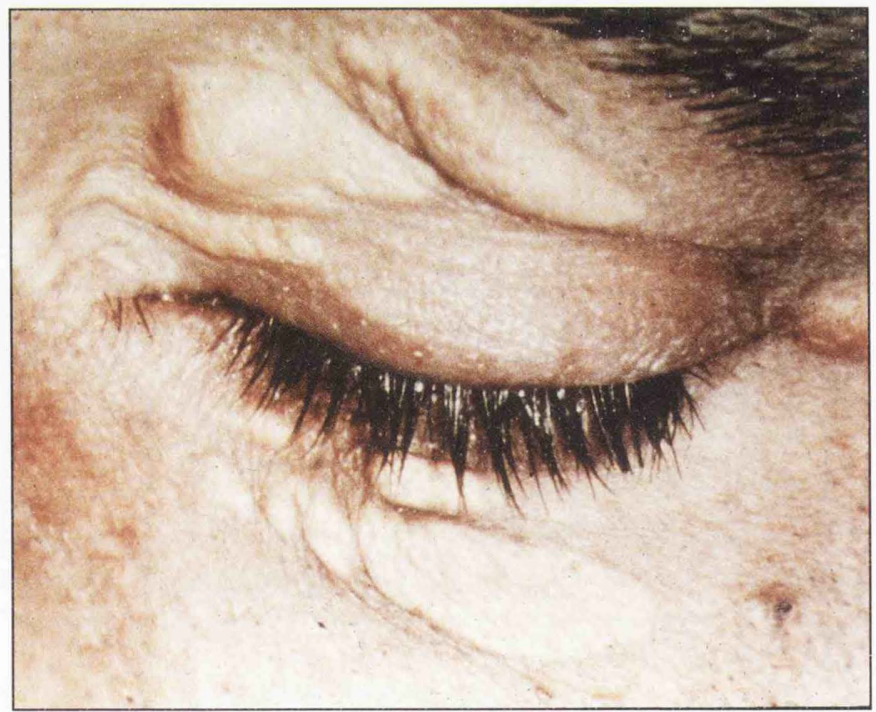

Figure 2 Xanthalesma: a clinical sign in dyslipidaemias

Source: $M S D$

\section{Case 5: a complex case}

A 47-year-old man presented with a high cholesterol and TG. He was asymptomatic and smoked 15 a day, drank 36 units of alcohol a week and was active on a low fat diet. There was a strong family history of cerebrovascular disease. On examination his BMI was 25 and BP $155 / 100$. His TSH and fasting glucose were found to be normal. 


$\begin{array}{lcccc}\text { TC } & \text { LDL } & \text { HDL } & \text { FTG } & \text { Risk } \\ 8.6 & * & 1.3 & 11.0 & 6.7 \\ 7.6 & * & 1.0 & 11.5 & 7.5 \\ 7.4 & * & 1.4 & 8.9 & 5.3 \\ 11.7 & * & 3.7 & 5.3 & 3.1\end{array}$

The statin was stopped and he was told to stop smoking, reduce weight by $7 \mathrm{lb}(3 \mathrm{~kg})$ and reduce alcohol consumption to 21 units a week. One month later:

\section{TC LDL HDL FTG Risk \\ $\begin{array}{lllll}12.2 & * & 3.2 & 6.6 & 3.8\end{array}$}

He met his weight target and stopped smoking. His BP was improved at $135 / 80$. He was put on a stronger statin and seen again in 2/12:

$\begin{array}{ccccc}\text { TC } & \text { LDL } & \text { HDL } & \text { FTG } & \text { Risk } \\ 6.3 & * & 0.8 & 15 & 7.8\end{array}$

A third-generation fibrate was added and within $2 / 12$ :

$\begin{array}{ccccc}\text { TC } & \text { LDL } & \text { HDL } & \text { FTG } & \text { Risk } \\ 5.3 & * & 1.1 & 5.7 & 4.8\end{array}$

In view of the family history of cerebrovascular disease, his plasma homocysteine was checked and found to be twice the normal upper limit. He was prescribed folic acid, $1200 \mathrm{mcg}$ daily.

In summary he has severe hypertriglyceridaemia. The apparent high HDL may not in his case afford protection because it is associated with alcohol ${ }^{(5)}$. This is a controversial issue. It is also possible that the HDL measurements on two occasions were inaccurate because of analytical interference by the high TG. The current plan is more weight reduction. Hopefully we will see his LDL soon as the FTG falls, though his apoB is now within the ref range. His apoE2 status is negative for remnant lipidaemia. ApoC2 deficiency is a possible diagnosis.

\section{DYSLIPIDAEMIA AND CHD RISK}

The most common type of dyslipidaemia is Familial Combined Hyperlipidaemia. Further investigation is necessary to determine whether it is primary or secondary to some other disorder. It is diagnosed in fasting serum and is the same phenotypically as the lipid abnormality in the 'Metabolic Syndrome' (Table 4).
The LDL increase is generally modest compared with heterozygous familial hypercholesterolaemia but the atheroma potential of this abnormality may exceed it.

The magnitude of coronary disease risk increases with each additional risk factor such as hypertension, cigarette smoking, impaired glucose tolerance and higher still in type 2 diabetes and also in diabetic nephropathy. The dyslipidaemia most characteristic of type 2 diabetes mellitus is of the same pattern. It develops many years before diabetes is diagnosed and, indeed, IHD may pre-date the onset of diabetes.

This abnormality may be caused by any of those factors in Table 1. These are all associated with accelerated atherogenesis. A key clinical feature is abdominal obesity with a waist circumference measured midway between the iliac crest and costal margin above 40 inches in men and 35 inches in women.

The strange thing is that medications which normalize this pattern are available but not generally used. The reason could be said to be three-fold:

- Policies follow intervention trials of statins in CHD which show clinical benefit. Eg $4 \mathrm{~S}^{(6)}$

- There have been safety concerns about fibrate drugs which are based on early trials with the obsolete drug clofibrate.(7)

- The fact is that fasting serum TG is an independent risk factor for CHD, which is not always appreciated ${ }^{(8)}$.

A meta-analysis of trials has shown a clinical benefit from TG reduction. Post hoc subgroup analysis of the Helsinki trial shows that in those with a high FTG and low HDL the reduction in cardiac events over 5 years was $71 \%{ }^{(9)}$.

The success of eicosanopentanoic acid (Omacor) as a plaque stabiliser post MI and its effects on reducing TG may well prove to be a therapeutic target as beneficial as lowering LDL below 3 and increasing HDL above 1.0. Targets for FTG in the US are presently <1.7; another value advocated is 2.3 but the lower figure is the value above which small dense

\section{Practice point}

FTG over 2.3 HDL less than 1.0 LDL over 3.5 is the commonest lipid abnormality in the general population and constitutes part of the atherogenic phenotype. It may be primary or a mixture of primary and secondary lipidaemias such as common hypercholesterolaemia with obesity or alcohol excess. (Table 4.)

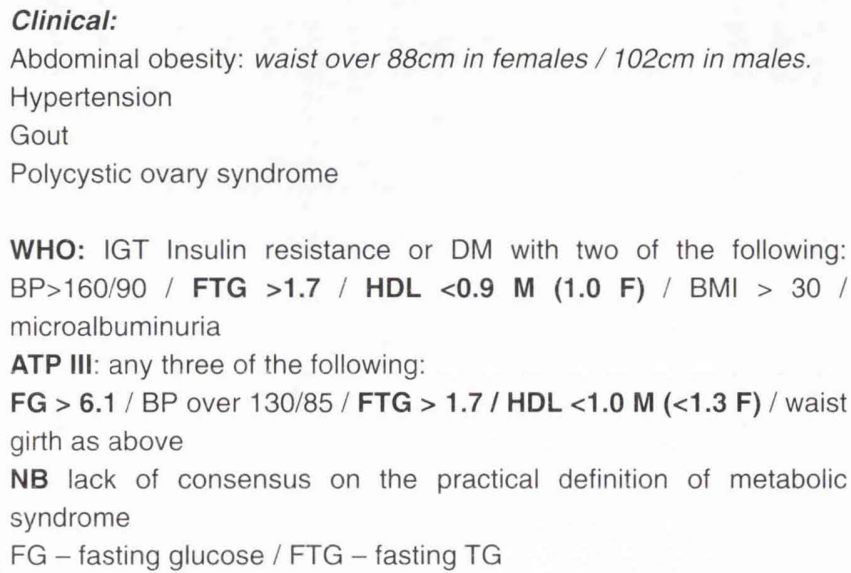

\section{Pathophysiological:}

Microalbuminuria

Insulin resistance

Low HDL - small and dense

Prolonged postprandial lipaemia

Small Dense LDL (LDL III)

Increased IDL

Increased NEFA flux

Increased non-receptor-mediated LDL catabolism

Hyperuricaemia

Reduced SHBG

Increased clotting factors: VII TBPA Fibrinogen

Glucose intolerance 
LDL predominates in ultracentrifuge profiles of LDL. Clarification of clinical outcomes in diabetes is awaited in the FIELD trial with fenofibrate.

Sacks et al found that the response to pravastatin in the CARE trial was highly significant in patients whose FTG was less than $1.6 \mathrm{mmol} / \mathrm{l}$ and reduced but not significantly in those with FTG greater than this value ${ }^{(10)}$.

There are many disorders of lipid metabolism and many are worthy of medical intervention.

\section{REFERENCES}

1. Betteridge J, Illingworth R, Shepherd J, (editors). The Disorders of Lipids and Lipoproteins. 1st ed. Arnold 1999.

2. Durrington P. The Hyperlipidaemias. 2nd ed. Butterworth Heinemann 1999.

3. Pyorala K, Wood D. Prevention of coronary heart disease in clinical practice. European recommendations revised and reinforced. Eur Heart J 1998;19(10):1413-5

4. Sacks FM, Expert Group on HDL Cholesterol. The role of high-density lipoprotein (HDL) cholesterol in the prevention and treatment of coronary heart disease: expert group recommendations. Am J Cardiol 2002; $90(2): 139-143$
5. Salonen JT. Liver damage and protective effect of high density lipoprotein cholesterol. Br Med J 2003;327:1082-3

6. Randomised trial of cholesterol lowering in 4444 patients with coronary heart disease: the Scandinavian Simvastatin Survival Study (4S). Lancet 1994;344:1383-9

7. A co-operative trial in the primary prevention of ischaemic heart disease using clofibrate. Report from the Committee of Principal Investigators. $\mathrm{Br}$ Heart $\mathrm{J}$ 1978;10:1069-118

8. Hokanson JE, Austin MA. Plasma triglyceride level is a risk factor for cardiovascular disease independent of high-density lipoprotein cholesterol level: a metaanalysis of population-based prospective studies. J Cardiovasc Risk 1996;3(2):213-9

9. Manninen V, Tenkanen L, Koskinen P, Huttenen JK, Manttari M, Heinonen OP et al. Joint effects of serum triglyceride and LDL cholesterol and HDL cholesterol concentrations on coronary heart disease risk in the Helsinki Heart Study. Implications for treatment. Circulation 1992;85:37-45

10. Sacks FM, Pfeffer MA, Moye LA, Rouleau JL, Rutherford JD, Cole TG et al. The effect of pravastatin on coronary events after myocardial infarction in patients with average cholesterol levels. Cholesterol and Recurrent Events Trial investigators. New Engl J Med 1996:335:1001-1009 